\title{
Análise do tempo de retirada do respirador artificial no paciente submetido a traqueostomia precoce e após sete dias de ventilação mecânica invasiva
}

\author{
Analysis of removal time of artificial ventilator in patients submitted to early tracheostomy, \\ after seven days of invasive mechanical ventilation \\ Análisis del tiempo de retiro del ventilador mecánico en pacientes sometidos a traqueotomía \\ temprana y después de siete dias de ventilación mecánica invasiva \\ Jonas Davi Heiderick Mota', Yuri de Souza Rodrigues², Flávia dos Santos Lugão de Souza³
}

RESUMO | A realização da traqueostomia precoce pode reduzir o tempo de ventilação do paciente, facilitando o desmame da prótese ventilatória. Além disso, reduz os custos de internação. Este estudo tem como objetivo analisar o tempo de retirada do respirador no paciente traquestomizado precocemente após sete dias de ventilação. Trata-se de pesquisa documental, retrospectiva, de carácter descritivo, sendo a coleta de dados realizada nos documentos de indicadores hospitalares de unidade de terapia intensiva de um hospital da Zona da Mata mineira. Foram coletados dados de 50 pacientes que obtiveram sucesso e preencheram os critérios de desmame da ventilação mecânica, entre homens e mulheres, com média de idade de 56,6 anos. Para análise estatística, os pacientes foram divididos em dois grupos: grupo precoce, de pacientes que realizaram traqueostomia com menos de sete dias de intubação orotraqueal; e grupo tardio, de pacientes que permaneceram por mais de sete dias com tubo orotraqueal até a realização da traqueostomia. Obtivemos um resultado significativo $(p=0,04)$ quanto aos dias em ventilação mecânica após a realização de traqueostomia, demonstrando que nos pacientes com traqueostomia precoce houve menos dias em ventilação mecânica invasiva do que nos pacientes com traqueostomia tardia. Foi constatado um resultado significativamente menor do tempo de ventilação dos pacientes traquestomizados precocemente quando comparados com a traqueostomia tardia. Ensaios clínicos randomizados de alta qualidade são necessários para avaliar melhor as possíveis diferenças da retirada da ventilação mecânica entre pacientes traqueostomizados.

Descritores | Traqueostomia; Desmame do Respirador.

ABSTRACT I Early tracheostomy can reduce patient ventilation time, facilitating the weaning process and reducing hospitalization costs. This study analyzes the removal time of ventilators in patients submitted to early tracheostomy, after seven days of ventilation. This is a documentary, retrospective, and descriptive research. Data were collected from documents with hospital indicators of an intensive care unit in the Zona da Mata region in Minas Gerais. Data consisted of 50 patients who were successful in the weaning of mechanical ventilation and met the study criteria. They were both men and women, with a mean age of 56.6 years. For statistical analysis, the patients were divided into two groups: an early group - patients who underwent tracheostomy with less than seven days of orotracheal intubation; and a late group - patients who remained for more than seven days with orotracheal tube before tracheostomy. We obtained a significant result ( $p=0.04$ ) regarding the days on mechanical ventilation after tracheostomy, demonstrating that patients with early tracheostomy remained fewer days on invasive mechanical ventilation than patients with late tracheostomy. A significantly lower result of ventilation time was observed in patients submitted to early compared with late tracheostomy. High-quality randomized clinical trials are

1Faculdade do Futuro - Manhuaçu (MG), Brasil. E-mail: jdheiderick1@gmail.com. Orcid: 0000-0001-8881-8752 ${ }^{2}$ Faculdade do Futuro. Manhuaçu (MG), Brasil. E-mail: ysrodrigues@hotmail.com. Orcid: 0000-0002-7851-6563

3Universidade Federal do Estado do Rio de Janeiro (Unirio) - Rio de Janeiro (RJ), Brasil. E-mail: flavia.I.s@terra.com. Orcid: 0000-0003-0041-4409 
needed to better evaluate the possible differences in mechanical ventilation withdrawal among tracheostomized patients. Keywords | Tracheostomy; Ventilator Weaning.

RESUMEN I La realización de traqueotomía temprana puede reducir el tiempo de ventilación del paciente, facilitando la desconexión del soporte ventilatorio. Además, reduce los costes de hospitalización. Este estudio tiene como objetivo analizar el tiempo de retiro del ventilador en los pacientes traqueostomizados tempranamente después de siete días de soporte ventilatorio. Se trata de una investigación documental, retrospectiva, descriptiva, que recopiló los datos de documentos hospitalarios de la unidad de cuidados intensivos de un hospital en Zona da Mata en Minas Gerais (Brasil). Se recogieron datos de 50 pacientes, entre hombres y mujeres con promedio de edad de 56,6 años, que tuvieron éxito y cumplieron los criterios para la desconexión del ventilador mecánico. Para el análisis estadístico, se dividieron a los pacientes en dos grupos: Grupo temprano, pacientes que se sometieron a traqueotomía con menos de siete días de intubación orotraqueal; y Grupo tardío, pacientes que permanecieron más de siete días con un tubo orotraqueal hasta la realización de la traqueotomía. Hubo un resultado significativo $(p=0,04)$ con respecto a los días de ventilación mecánica tras la realización de traqueotomía, lo que demuestra que los pacientes con traqueotomía temprana pasaron menos días con ventilador mecánico invasivo que los pacientes con traqueotomía tardía. Se encontró un resultado significativamente menor del tiempo de ventilación de los pacientes con traqueostomía temprana en comparación a los pacientes con traqueotomía tardía. Se necesitan ensayos clínicos aleatorios de alta calidad para evaluar mejor las posibles diferencias en el retiro del ventilador mecánico entre los pacientes traqueostomizados. Palabras clave | Traqueotomía; Desconexión del Ventilador.

\section{INTRODUÇÃO}

As indicações para a realização da traqueostomia são claras, incluindo ventilação mecânica prolongada, necessidade de proteção das vias aéreas, dificuldade de desmame da ventilação mecânica, escore de coma de Glasgow persistente $<8$, polineuropatia e obstrução das vias aéreas superiores. Pacientes internados em unidade de terapia intensiva (UTI) apresentam cada vez mais frequente, doenças pulmonares crônicas, neurológicas e degenerativas além das comorbidades associadas ${ }^{1}$.

O uso da traqueostomia precoce em pacientes eletivos a ventilação mecânica prolongada pode ajudar a reduzir a mortalidade hospitalar de forma significativa ${ }^{2,3}$.

Assim que o problema de base do paciente em ventilação mecânica for resolvido inicia-se o processo de retirada da respiração artificial, sendo avaliado diariamente pelo profissional responsável o momento certo da extubação ou retirada da prótese ventilatória. $\mathrm{O}$ paciente que apresentar um prognóstico prolongado estará sujeito a um tempo maior de uso da ventilação mecânica invasiva, a maioria dos pacientes com este prognóstico é submetido a cirurgia de traqueostomia, ao substituir um tubo endotraqueal por um tubo de traqueostomia você reduz o trabalho respiratório, melhora a depuração da secreção das vias aéreas, diminui o risco de pneumonia associada a ventilação mecânica, permite redução da sedação, facilita o processo de desmame e alivia o trabalho respiratório. Atualmente, são utilizadas duas técnicas cirúrgicas: aberta e percutânea, a técnica cirúrgica percutânea à beira do leito é cada vez mais utilizada na realização da traqueostomia e é considerada um procedimento seguro e de baixo custo ${ }^{1,4,5}$.

Não se sabe ao certo o tempo ideal para a realização da traqueostomia de forma que possa trazer benefícios como: a melhora da fisiologia respiratória, diminuição do uso de sedativos e aumento da mobilidade. Isto se deve a potenciais malefícios que devem ser destacados como por exemplo o risco do procedimento cirúrgico e o índice de mortalidade a curto ou longo prazo, além da nossa incapacidade de prever com precisão quais pacientes necessitarão de ventilação mecânica prolongada. De acordo com Mehta et al. ${ }^{6}$, atrasar a decisão de realizar a traqueostomia pode permitir que pacientes com alto risco de ventilação mecânica prolongada sejam liberados do ventilador e evitar traqueostomia desnecessária ${ }^{6}$.

Alguns estudos apontam que a realização da traqueostomia precoce não influencia o índice de mortalidade em pacientes submetidos a ventilação mecânica prolongada ${ }^{2,6-10}$.

A diretriz brasileira de ventilação mecânica ${ }^{11}$ institui que o procedimento de traqueostomia precoce seja em até 7 dias de ventilação e realizado em pacientes com traumatismo raquimedular cervical alto, traumatismo crânio encefálico com Glasgow < 8 e estimativa de ventilação prolongada, nos demais pacientes aguardar até 14 dias ${ }^{11}$.

Como visto, estudos abordam os benefícios da realização precoce da traqueostomia influenciando na retirada precoce do respirador artificial. O presente estudo tem como objetivo avaliar se há diferença no tempo de 
ventilação mecânica em pacientes traqueostomizados precocemente e após 7 dias de ventilação mecânica em um Hospital da Zona da Mata Mineira.

\section{METODOLOGIA}

O projeto foi aprovado pelo Comitê de Ética em Pesquisa com Seres Humanos (CEP) da instituição Sociedade de Ensino Superior de Manhuaçu Ltda., com CAAE no15394619.3.0000.5106. Após aprovação pelo CEP, foi iniciada uma pesquisa documental, retrospectiva de agosto de 2018 a setembro 2019, de carácter descritivo, sendo a coleta de dados realizada nos documentos de indicadores hospitalares da UTI de um hospital da Zona da Mata mineira.

\section{Sujeitos da amostra}

A pesquisa foi realizada com todos os pacientes que preencheram critérios de desmame da ventilação mecânica invasiva e foram retirados com sucesso do respirador artificial no período de agosto de 2018 a setembro de 2019.

\section{Instrumentos de coleta de dados}

O instrumento para a coleta de dados utilizado no estudo é um documento padrão que faz parte do Procedimento Sistêmico PRS.EMA.009 para admissão e controle do desmame da ventilação mecânica invasiva do hospital em questão. A liberação destes dados para pesquisa é realizada através da autorização da direção e coordenação da UTI.

\section{Teste de Respiração Espontânea (TRE)}

São avaliados os pacientes 3 vezes ao dia (manhã e tarde e noite) os que preencherem os critérios de reversão da insuficiência respiratória devem ser submetidos a um TRE, que deve ser feito colocando uma pressão de suporte ventilatório (PSV) de 5 a $7 \mathrm{cmH}_{2} 0$ por 30 a 120 minutos, se o teste for positivo, colhe-se gasometria arterial, parâmetros normais, é decidido a retirada da ventilação mecânica.

\section{Tratamento estatístico dos dados}

Os dados coletados foram digitados no programa Excel for Windows 10 (Microsoft Office Professional Plus 2016) e, posteriormente avaliados pelos autores com as médias e desvio-padrão. Também foi realizado o teste $t$ de Student nas amostras e o nível de significância estatística adotado para este teste será de $5 \%$, ou seja, o valor de $P$ igual ou inferior a 0,05 para resultados estatisticamente significativos $(\mathrm{p}<0,05)$.

\section{RESULTADOS}

Foram coletados dados de 50 pacientes que obtiveram sucesso e preencheram os critérios de desmame da ventilação mecânica invasiva, sendo 28 do sexo feminino (56\%) e 22 do sexo masculino (44\%). Para análise estatística, os pacientes foram divididos em dois grupos distintos: grupo tardio, de pacientes que permaneceram por mais de sete dias com tubo orotraqueal até a realização da traqueostomia; e grupo precoce, de pacientes que realizaram traqueostomia até sete dias de intubação orotraqueal (Tabela 1). No total o grupo tardio permaneceu em média 16,57 dias em ventilação mecânica invasiva e o grupo precoce 8,07 dias.

A amostra do grupo tardio foi de 23 pacientes, sendo 12 do sexo feminino $(52,17 \%)$ e 11 do sexo masculino (47,83\%). A média de idade foi de 56,67 19,9 anos. Em média a permanência de dias com tubo orotraqueal foi de 10,87, e a permanência em ventilação mecânica invasiva após a realização da traqueostomia foi de 5,70 dias.

A amostra do grupo precoce foi de 27 pacientes, sendo 16 do sexo feminino $(59,25 \%)$ e 11 do sexo masculino (40,75\%). A média de idade foi de 66,29 16,98 anos. Em média a permanência de dias com tubo orotraqueal até a realização da traqueostomia foi de 4,7 dias, e a permanência em ventilação mecânica invasiva após a realização da traqueostomia foi de 3,37 dias.

Tabela 1. Dados epidemiológicos coletados nos dois grupos deste estudo

\begin{tabular}{lrrr} 
& \multicolumn{3}{c}{$\mathrm{n}(\%)$ ou média \pm DP } \\
\cline { 2 - 4 } Variáveis & Grupo tardio & Grupo precoce & Valor de p \\
Número de pacientes & $23(46 \%)$ & $27(54 \%)$ & \\
Idade & $56,67 \pm 19,9$ & $66,29 \pm 16,98$ & 0,09 \\
Dias em VM antes TQT & $10,87 \pm 2,36$ & $4,70 \pm 1,77$ & \\
Dias em VM após TQT & $5,70 \pm 3,86$ & $3,37 \pm 3,92$ & ${ }^{*} 0,04$ \\
Alta & $11(48 \%)$ & $13(48 \%)$ & 0,9 \\
Óbito & $12(52 \%)$ & $14(52 \%)$ & \\
Sexo & & & \\
Feminino & $12(52 \%)$ & $16(59 \%)$ & 0,62 \\
Masculino & $11(48 \%)$ & $11(41 \%)$ & \\
Motivo IOT & & & \\
Neurológica & $11(48 \%)$ & $10(37 \%)$ & \\
Pulmonar & $8(35 \%)$ & $11(40 \%)$ & 0,8 \\
Cardíaca & $1(4 \%)$ & $4(15 \%)$ & \\
Renal & $1(4 \%)$ & $1(4 \%)$ & \\
Apache II & $13 \pm 4,51$ & $17 \pm 4,7$ & $* 0,004$
\end{tabular}

DP: desvio-padrão; VM: ventilação mecânica; TQT: traqueostomia; IOT: intubação orotraqueal. *Valor estatisticamente significativo. 
Não observamos diferença de dados com valores significativos nos dois grupos estudados quanto a idade, número de altas/óbitos, gênero e diagnóstico.

A mediana e desvio-padrão dos escores Apache II no grupo precoce foi de $17 \pm 4,7$ e no grupo tardio $13 \pm 4,51$.

Para análise entre os grupos quanto ao fator causal da intubação orotraqueal, separamos em 4 subgrupos de acordo com a função fisiológica acometida: Neurológica, renal, pulmonar e cardíaca, o teste t de Student foi calculado de acordo com o total de pacientes por função acometida, portanto com distribuição bicaudal e variação desigual de duas amostras. Os dados quanto à quantidade de pacientes para cada tipo de diagnóstico estão na Tabela 2.

Tabela 2. Quantidade de pacientes e diagnósticos que levaram à intubação orotraqueal

\begin{tabular}{|c|c|c|}
\hline \multicolumn{3}{|c|}{ Função acometida e diagnóstico } \\
\hline \multicolumn{3}{|c|}{ Grupo tardio } \\
\hline Função acometida & № Pacientes & Diagnóstico \\
\hline \multirow[t]{3}{*}{ Neurológica } & 8 & AVE \\
\hline & 3 & TCE \\
\hline & 1 & Encefalopatia \\
\hline Renal & 1 & IRC \\
\hline \multirow[t]{2}{*}{ Pulmonar } & 6 & PNM \\
\hline & 2 & IRPA \\
\hline \multirow{2}{*}{ Cardíaca } & 1 & EAP \\
\hline & 1 & ICC \\
\hline \multicolumn{3}{|c|}{ Grupo precoce } \\
\hline \multirow[t]{5}{*}{ Neurológico } & 4 & AVE \\
\hline & 3 & TCE \\
\hline & 1 & Encefalopatia \\
\hline & 1 & ELA \\
\hline & 1 & Edema cerebral \\
\hline Renal & 1 & IRC \\
\hline \multirow[t]{5}{*}{ Pulmonar } & 4 & PNM \\
\hline & 4 & IRPA \\
\hline & 2 & DPOC \\
\hline & 1 & Fibrose pulmonar \\
\hline & 1 & Derrame pleural \\
\hline \multirow[t]{2}{*}{ Cardíaca } & 3 & ICC \\
\hline & 1 & BAVT \\
\hline
\end{tabular}

AVE: acidente vascular encefálico; TCE: traumatismo crânio encefálico; IRC: insuficiência renal crônica; PNM: pneumonia; IRPA: insuficiência respiratória pulmonar aguda; EAP: edema agudo pulmonar: ICC: insuficiência cardíaca congestiva; ELA: esclerose lateral amiotrófica; DPOC: doença pulmonar obstrutiva crônica; BAVT: bloqueio átrio ventricular; Sida: síndrome da imunodeficiência adquirida.

\section{DISCUSSÃO}

Quando comparamos a média dos dias de permanência em ventilação mecânica invasiva após a realização de traqueostomia entre os dois grupos, obtivemos um resultado demonstrando que nos pacientes com traqueostomia precoce os dias em ventilação mecânica invasiva foi significativamente $(\mathrm{p}=0,04)$ menor que nos pacientes com traqueostomia tardia.

Em 2011, Ferreira e Cavenaghi ${ }^{10}$, em São Paulo, em um artigo de revisão buscando avaliar a traqueostomia precoce no desmame da ventilação mecânica, chegaram à conclusão de que a realização da traqueostomia precoce pode reduzir a mortalidade, tempo de internação em UTI e tempo de ventilação mecânica. Ainda segundo os autores, estava havendo divergências acerca da definição dos conceitos de traqueostomia precoce; porém, as vantagens e desvantagens da técnica pareciam estar bem elucidadas e com boas evidências para a prática clínica ${ }^{10}$.

Um estudo de revisão publicado em 2016 com oito ensaios clínicos randomizados totalizando 1.977 pacientes críticos concluiu que as traqueostomias precoces podem ser preferenciais às traqueostomias tardias e devem ser realizadas antes de 10 dias, quando se espera que o paciente exija ventilação mecânica a longo prazo (>21 dias) devido a seus benefícios quando realizada de forma precoce ${ }^{7}$.

Em uma coleta de dados feita no ano de 2017 em Taiwan, com uma amostra de 401 pacientes traqueostomizados, Huang et al. ${ }^{2}$ observaram que a traqueostomia precoce (14 dias, neste caso) pode estar associada a resultados favoráveis de pacientes críticos em ventilação mecânica prolongada. Foram encontrados valores significativos quanto a mortalidade hospitalar e risco de pneumonia associado a ventilação mecânica ${ }^{2}$.

Outra revisão sistemática da plataforma Cochrane foi realizada em 2015 por Andriolo et al. ${ }^{9}$ com o objetivo de avaliar a eficácia e segurança da traqueostomia precoce versus a traqueostomia tardia. Mostra-se os potenciais benefícios, embora não de forma definitiva, da traqueostomia precoce em relação a tardia para mortalidade, tempo de permanência na UTI e duração da ventilação mecânica. Além disso relata menores probabilidades de pneumonia e maior sucesso de desmame, ressaltamos ainda que neste estudo de Andriolo et al. foi estabelecido como traqueostomia precoce quando realizada em até 10 dias de intubação orotraqueal e tardia com mais de 10 dias de intubação 9 .

Há, portanto, indícios na literatura vigente que corroboram o valor significativo encontrado neste estudo quanto aos dias de ventilação mecânica após o procedimento de traqueostomia.

No entanto, ressalta-se que as evidências disponíveis devem ser consideradas com cautela, pois as informações são insuficientes para permitir conclusões sobre qualquer 
subgrupo ou característica individual potencialmente associada às melhores indicações para traqueostomia precoce ou tardia, A revisão de Keeping ${ }^{7}$, por exemplo, mostra que em duas metanálises estudadas que avaliaram o tempo de ventilação mecânica não relataram diferença significativa entre os grupos traqueostomia precoce e tardia ${ }^{7}$.

Em 2015, em um estudo de coorte retrospectivo com 70 pacientes cardiológicos de uma UTI no sul do Brasil, Sakae et al. verificaram que a traqueostomia precoce parece não estar associada à redução da mortalidade ${ }^{8}$.

Mehta et al. ${ }^{6}$, em 2016, ao observar que as taxas de traqueostomia precoce têm aumentado nos Estados Unidos em pacientes com risco de ventilação mecânica prolongada, realizaram um estudo com uma amostra populacional significativa de pacientes hospitalizados, tendo como objetivo avaliar a utilização e os desfechos separadamente entre os pacientes com as duas indicações mais comuns de traqueostomia - trauma e pneumonia/sepse. Para esta pesquisa foi utilizada a Agência Nacional de Pesquisa e Qualidade em Assistência à Saúde dos EUA de 2012. Com uma amostra probabilística estratificada de todas as internações não federais, identificou dados de reclamações administrativas extrapolados de $20 \%$ de todas as altas de mais de 4 mil hospitais em 44 estados. Foi investigada a variação no nível hospitalar da utilização precoce de traqueostomia e os resultados dos pacientes associados à traqueostomia precoce. Observaram uma ampla variação na utilização da traqueostomia precoce intra-hospitalar e chegaram à conclusão que a mortalidade não diferiu com base no tempo de traqueostomia para trauma ou pneumonia/sepse ${ }^{6}$.

A revisão de Keeping ${ }^{7}$ também conclui que não há diferença significativa na mortalidade entre traqueostomia precoce e tardia em pacientes críticos ${ }^{7}$.

Estes dados encontrados em alguns estudos referentes ao índice de mortalidade entre os grupos de traqueostomia precoce e tardia entram em concordância com nosso estudo já que não obtivemos dados significativos quanto ao número de alta e óbitos entre os grupos estudados ${ }^{2,6-10}$.

Obtivemos um resultado significativo $(\mathrm{p}=0,004)$ quando comparamos a média do escore Apache II entre os dois grupos. No grupo precoce a média do escore e respectivo desvio-padrão foi de $17 \pm 4,7$ e no grupo tardio $13 \pm 4,51$. Portanto a estimativa de mortalidade nos pacientes traqueostomizados de forma precoce é significativamente maior que no grupo em que a traqueostomia foi realizada após sete dias de intubação orotraqueal. O que sugere que os pacientes mais graves normalmente são os eletivos ao procedimento de traqueostomia de forma precoce.
Considerando que o grupo precoce teve em média um escore Apache II maior e mesmo assim, permaneceu por menos tempo em ventilação mecânica, e que não obtivemos um resultado significativo para o número de altas/óbitos entre os dois grupos, podemos concluir que o risco de óbito não influencia no tempo de ventilação mecânica.

Este dado corrobora um estudo de coorte realizado por Aranha et al. ${ }^{12}$ com 190 pacientes em um hospital de São Paulo, em 2007, no qual se buscou comparar a efetividade da traqueostomia quanto ao tempo em que foi realizada. O estudo ainda concluiu que quanto ao período de realização da traqueostomia, apesar de muitos autores demonstrarem que a traqueostomia precoce se encontra associada à redução do tempo de ventilação mecânica e do tempo de internação na UTI. Não foram encontrados dados significativos quanto a estas vantagens. A média de dias de intubação orotraqueal do grupo com traqueostomia precoce foi de 10,4 $\pm 1,54$ e a média do grupo de traqueostomia tardia foi de $16,23 \pm 1,95$. Em nosso estudo os dias de permanência com tubo orotraqueal no grupo com traqueostomia tardia foi de $10,87 \pm 2,36$, ou seja, podemos comparar o nosso grupo de traqueostomia precoce com o grupo de traqueostomia tardia deste estudo no tocante a dias de intubação orotraqueal ${ }^{12}$.

O tempo médio de duração da ventilação mecânica após a realização da traqueostomia nos pacientes do grupo precoce do estudo de Aranha foi de 29,73 $\pm 19,1$, em nosso estudo este tempo no grupo que se equivale ao tempo de intubação orotraqueal (grupo tardio) foi de 5,70 3 , 86 . Podemos associar essa discrepância ao corte temporal de ambos os estudos, evidenciando a importância de estudos voltados para esta área e a constante e rápida evolução dos recursos e cuidados assistenciais ao paciente crítico e seu desmame da ventilação mecânica invasiva.

\section{CONCLUSÃO}

Foi identificado que nos pacientes com traqueostomia precoce os dias em ventilação mecânica invasiva foi significativamente $(p=0,04)$ menor que nos pacientes com traqueostomia tardia. Em concordância com a literatura mais recentemente pesquisada, nosso estudo ressalta não ter relação a realização da traqueostomia precoce com a taxa de mortalidade.

Os resultados devem ser analisados cautelosamente, as informações são insuficientes para permitir conclusões relevantes associadas às melhores indicações para 
traqueostomia precoce ou tardia, pois a heterogeneidade clínica é uma característica dos pacientes em UTI geral. São necessarias pesquisas de ensaios clínicos randomizados adicionais de alta qualidade para avaliar melhor as possíveis diferenças na retirada da ventilação mecânica entre pacientes submetidos a traqueostomia precoce e tardia em pacientes críticos. Assim como critérios para determinar qual o tempo exato para ser considerado traqueostomia precoce, devido a variação de dias na metodologia estudada.

\section{REFERÊNCIAS}

1. Zapata-Contreras L, Hoyos-Cuervo CE, Florián-Pérez MC. Open tracheostomy in patients with dual platelet aggregation inhibitors: case series. Rev Colomb Anestesiol. 2019;47(3):189-93. doi: 10.1097/cj9.0000000000000113

2. Huang C-T, Lin J-W, Ruan S-Y, Chen C-Y, Yu C-J. Preadmission tracheostomy is associated with better outcomes in patients with prolonged mechanical ventilation in the postintensive care respiratory care setting. J Formos Med Assoc. 2017;116(3):169-76. doi: 10.1016/j.jfma.2016.05.005

3. Avalos N, Cataldo R, Contreras L. Unassisted percutaneous tracheostomy: a new flow chart decision making based on simple physical conditions. Am J Otolaryngol. 2019;40(1):57-60. doi: 10.1016/j.amjoto.2018.11.001

4. Lim C-K, Ruan S-Y, Lin F-C, Wu C-L, Chang H-T, Jerng J-S, et al. Effect of tracheostomy on weaning parameters in difficultto-wean mechanically ventilated patients: a prospective observational study. PLOS ONE. 2015;10(9):e0138294. doi: 10.1371/journal.pone.0138294

5. Budweiser S, Baur T, Jörres RA, Kollert F, Pfeifer M, Heinemann F. Predictors of successful decannulation using a tracheostomy retainer in patients with prolonged weaning and persisting respiratory failure. Respiration. 2012;84(6):469-76. doi: 10.1159/000335740

6. Mehta AB, Cooke CR, Wiener RS, Walkey AJ. Hospital variation in early tracheostomy in the United States: a population-based study. Crit Care Med. 2016;44(8):1506-14. doi: 10.1097/CCM.0000000000001674

7. Keeping A. Early versus late tracheostomy for critically ill patients: a clinical evidence synopsis of a recent Cochrane Review. Can J Respir Ther. 2016 [cited 2020 Nov 12];52(1):27-28. Available from: https://www.ncbi.nlm.nih.gov/pubmed/26909011

8. Sakae TM, Sakae GRFM, Schmitz RL, Sakae DY. Comparing mortality in early and late tracheostomy in cardiologic patients from an intensive care unit in south Brazil. ACM Arq Catarin Med. 2015;45(1):3-12.

9. Andriolo BNG, Andriolo RB, Saconato H, Atallah ÁN, Valente O. Early versus late tracheostomy for critically ill patients. Cochrane Database Syst Rev. 2015;(1):CD007271. doi: 10.1002/14651858. CD007271.pub3

10. Ferreira LL, Cavenaghi OM. Traqueostomia precoce no desmame da ventilação mecânica. Rev Bras Clin Med. 2011;9(6):432-6.

11. Associação de Medicina Intensiva Brasileira; Sociedade Brasileira de Pneumologia e Tisiologia. Diretrizes brasileiras de ventilação mecânica. São Paulo: Amib; 2013.

12. Aranha SC, Mataloun SE, Moock M, Ribeiro R. Estudo comparativo entre traqueostomia precoce e tardia em pacientes sob ventilação mecânica. Rev Bras Ter Intensiva. 2007;19(4):444-9. doi: 10.1590/S0103-507X2007000400007 